\title{
Prof. Attenberger im Wissenschaftlichen Beirat der Bundesärztekammer
}

Prof. Dr. Ulrike Attenberger, Direktorin der Klinik für Radiologie am Universitätsklinikum Bonn (UKB), wurde in den Wissenschaftlichen Beirat der Bundesärztekammer berufen. Sie wird damit künftig das Fachgebiet Radiologie in dem interdisziplinären Beratungsgremium vertreten.

Der Wissenschaftliche Beirat der Bundesärztekammer ist interdisziplinär aus Wissenschaftlern verschiedener Fachgebiete der Medizin zusammengesetzt. Seine Aufgabe ist die Beratung des Vorstands der Bundesärztekammer zu medizinisch-wissenschaftlichen Fragen, die insbesondere bei der Vorbereitung und Durchführung von Gesetzen und der ärztlichen Berufsausübung auftreten. Das Plenum des Wissenschaftlichen Beirats tritt zweimal jährlich zusammen.
Die 40-Jährige, die Forschungsaufenthalte in Harvard, Zürich und Wien absolviert hat, wird den Wissenschaftlichen Beirat der Bundesärztekammer ab sofort mit ihrer Expertise im Fachgebiet Radiologie mit dem Schwerpunkt der Tumordiagnostik bereichern. „Derzeit arbeiten wir daran, u. a. mithilfe von künstlicher Intelligenz (KI) anhand von Bilddaten besser voraussagen zu können, wie ein Patient auf die geplante Behandlung anspricht und zukünftig auch genauere Überlebensprognosen stellen zu können. Durch die Berufung in den Wissenschaftlichen Beirat erhoffe ich mir, die aktuellen Entwicklungen im Bereich der bildgebenden Diagnostik gewinnbringend für alle Fachdisziplinen für eine optimierte Patientenversorgung voranbringen zu können“, sagt Prof. Attenberger. Für die nächsten drei Jahre wird sie nun im Wissenschaftlichen Beirat beratend tätig sein.

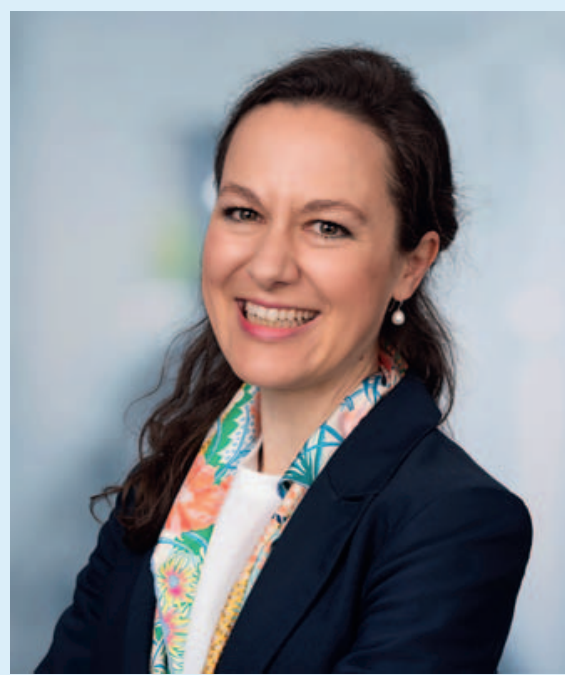

Prof. Dr. Ulrike Attenberger, Direktorin der Klinik für Radiologie am Universitätsklinikum Bonn (UKB), (c) Universitätsklinikum Bonn (UKB)/A. Winkler. 